\title{
El proceso de Toma de Decisiones en pacientes con Demencia tipo Alzheimer
}

\author{
José R. Alameda ${ }^{1}$, María P. Salguero ${ }^{1}$, Ana Merchán ${ }^{1}$, \\ Carmen $\mathrm{M}^{\mathrm{a}}$ Domínguez ${ }^{2}$ y Eva $\mathrm{M}^{\mathrm{a}}$ Rodríguez ${ }^{3}$ \\ ${ }^{1}$ Universidad de Huelva; ${ }^{2}$ Asociación de Familiares de Enfermos de Alzheimer y otras \\ Demencias de Huelva y Provincia; ${ }^{3}$ Federación Provincial de AFAs de Huelva
}

Tomar decisiones es común en nuestra vida diaria y contribuye a la adaptación a nuestro entorno. Es importante el conocimiento de los procesos de toma de decisiones y las posibles disfunciones debido a su deterioro. Los pacientes con demencia tipo Alzheimer (DTA) pueden tener alteraciones en la toma de decisiones ya en las primeras etapas, sobre todo en situaciones de incertidumbre. Estos procesos han sido analizados a partir de diferentes teorías como la hipótesis del marcador somático, con la IGT como una de las tareas más utilizadas. El objetivo de este estudio es analizar el proceso de toma de decisiones en pacientes con EA, y evaluar los tipos de decisiones tomadas en situaciones de incertidumbre con una modificación de la IGT, así como comprobar si las respuestas presentan tendencia al riesgo o no, y la relación entre el rendimiento y el estado cognitivo en cada una de las áreas evaluadas con el mini examen cognoscitivo, la prueba del dibujo del reloj (TRC-TRO), y la prueba de la fluidez verbal de Isaacs. Nuestros resultados muestran que el rendimiento del grupo DTA no es la óptima, ni en la puntuación global, ni en el análisis de cada uno de los bloques de la prueba, y sugiere una estrategia aleatoria en el proceso de elegir de las cartas, sugieren una tendencia al riesgo, aunque su ejecución muestra una cierta capacidad de aprender a lo largo de los bloques.

Palabras clave: Alzheimer, marcador somático, toma de decisiones, deterioro cognitivo.

The Decision Making process in patients with Alzheimer dementia type. Making decisions is common in our daily lives and contributes to the adaptation to our environment. That is why is relevant to know the decisions-making processes and the possible dysfunctions due to the deterioration of these processes. The patients with Alzheimer dementia (AD) can have alterations in decisions-making in early stages, particularly in situations of uncertainty. These processes have been analyzed from different theories, although the somatic marker hypothesis, as well as the related IGT, is one of the most used. The aim of this study is to analyze the process of making decisions in $\mathrm{AD}$ patients, and evaluate the types of choices made under situations of uncertainty using a modified IGT, as well as check if the AD group's responses are risk trends or not, and the relation between the performance of this group and the cognitive status in each of the areas evaluated with the cognitive mini-exam, the clock drawing test (TRC-TRO), and the Isaacs' Set Test of Verbal Fluency. Our results show that the performance of the AD group is not optimal, nor in the overall score or in the analysis of each sets of the test, and suggest a random strategy in the process of choose cards, suggest a tendency to risk, although the performances show some ability to learn along the sets.

Key words: Alzheimer, somatic marker, making decisions, cognitive impairment.

Correspondencia: Jose Ramón Alameda Bailén. Facultad de Psicología. Universidad de Huelva, Campus de El Carmen. Avenida de las Fuerzas Armadas, s/n. C.P. 21071. Huelva (España). E-mail: alameda@uhu.es 
La demencia es una problemática cada día más frecuente, entre ellas la enfermedad de Alzheimer corresponde al 50-60\% de los diagnósticos. Esto es debido al aumento de la esperanza de vida y la disminución de la natalidad, lo que implica un envejecimiento cada vez mayor de la población.

El diagnóstico de la enfermedad de Alzheimer (EA) presenta multitud de dificultades, ya que, la enfermedad presenta en sus inicios un proceso lento, progresivo y muy sutil que hace difícil su diagnóstico diferencial con el envejecimiento normal y con otras entidades clínicas. Además los síntomas se presentan de forma distinta según las áreas implicadas, la fase evolutiva y los niveles de gravedad. Los síntomas a nivel general, se dividen en tres ámbitos; área cognitiva que implica alteraciones de las capacidades mentales, a nivel funcional que representa una pérdida progresiva de la autonomía y a nivel psicológico y comportamental, presentando síntomas como ansiedad, depresión, alucinaciones, etc.

En el ámbito neuropsicológico en los pacientes con EA los deterioros más habituales se determinan en la memoria, la orientación, las capacidades verbales, la función visuoespacial y las funciones ejecutivas. Las funciones ejecutivas (FE) se han definido como los procesos que asocian ideas, movimientos y acciones simples y los orientan a la resolución de conductas complejas (Shallice, 1982), y se les atribuye un papel importante en actividades como la creatividad, en las operaciones formales del pensamiento, en la conducta social, en la toma de decisiones y en el juicio ético y moral (Price, Daffner, Stowe y Mesulam, 1990; Tirapu-Ustárroz, Muñoz-Céspedes y PelegrínValero, 2002). Con respecto, a la toma de decisiones, desde los años 90 surge la denominada Hipótesis del Marcador Somático (Damasio, 1994) que postula la relación de algunas regiones de la corteza prefrontal y el proceso de toma de decisiones (Damasio, Tranel y Damasio, 1990). Esta hipótesis pretende explicar por qué pacientes con daño frontal, con un funcionamiento adecuado en diversas áreas como memoria de trabajo, atención, lenguaje y capacidad intelectual presentaban un funcionamiento caótico a nivel personal y social. Desde esta teoría se defiende la relación existente entre el lóbulo frontal, la toma de decisiones y la emoción (Bechara, Damasio y Damasio, 2000). La emoción juega un papel principal en el proceso de toma decisiones en situaciones ambiguas y complejas. Ante situaciones con multitud de elecciones, la razón sería incapaz en un tiempo limitado de calcular los costes y beneficios de cada una de ellas, sería por tanto, la emoción por medio de los denominados "marcadores somáticos" o señales corporales la implicada en guiar hacia las buenas elecciones para obtener una toma de decisión acertada. En los pacientes, con un funcionamiento inadecuado a nivel social y personal, existe una ausencia a nivel emocional, observado mediante la ausencia de respuestas psicofisiológicas antes las elecciones desventajosas (Anderson, Bechara, Damasio, Tranel y Damasio, 2002; Bechara, Damasio, Tranel y Damasio, 1997; Bechara, Tranel, Damasio y Damasio, 1996; Tomb, Hauser, Deldin y Caramazza, 2002). 
Para estudiar la toma de decisión en el laboratorio Bechara, Damasio, Damasio y Anderson (1994) crearon la Iowa Gambling Task (IGT) que consta de 4 montones de cartas (A B C D) dos mazos ventajosos y dos desventajosos, con diferentes recompensas y castigos que el sujeto desconoce, ocultando una estrategia que convierte la prueba en una situación de incertidumbre.

En la actualidad la toma de decisiones se evalúa en multitud de pacientes; con esclerosis múltiple (García-Molina, Rodríguez, Vendrell, Junqué y Roig-Rovira, 2008; Kleeberg et al., 2004), esquizofrénicos (Bark, Dieckmann, Bogerts y Northoff, 2005; Ritter, Meador-Woodruff y Dalack, 2004); obsesivos-compulsivos (Cavedini et al., 2002; Cavedini et al., 2012) jugadores patológicos (De Ruiter, Oosterlaan, Veltman, van den Brink y Goudriaan, 2012; Goudriaan, Oosterlaan, de Beurs y van den Brink, 2005), pacientes neurológicos (Bechara et al., 1994, 2000; Clark, Manes, Antoun, Sahakian y Robbins, 2003; García-Molina et al., 2007) y consumidores de drogas (Dante, 2006; Grant, Contoreggi y London, 2000; Mazas, Finn y Steinmetz, 2000; Petry, Bickel y Arnett, 1998; Verdejo, Aguilar de Arcos y Pérez-García, 2004).

Los estudios realizados con demencia tipo Alzheimer son escasos (Delazer, Sinz, Zamarian y Benke, 2007; Moreno y Alameda, 2011; Zamarian, Weiss y Delazer, 2011) y con una heterogeneidad en las pruebas utilizadas ; la IGT, la Game of Dice Task o la Probability-Associated Gambling Task (PAG). Pero los resultados muestran que pacientes con EA presentan un deterioro en la toma de decisiones comparados con sujetos sanos, estos pacientes presentan una tendencia al riesgo, pero no tan pronunciada como en pacientes neurológicos.

El objetivo del presente trabajo es determinar el rendimiento o ejecución en la toma de decisiones en pacientes con diagnóstico Alzheimer en fase inicial y comparar con sujetos sanos, igualados en nivel educativo y edad. Se evalúa mediante un juego de Cartas, basado en la original IGT, pero con modificaciones en relación al total de jugadas, para disminuir el tiempo de ejecución, y en la cantidad de dinero, tanto en las ganancias como en las pérdidas, que aparece en cada carta.

\section{METODO}

\section{Participantes}

El grupo de pacientes en fase de inicio de la enfermedad de Alzheimer está compuesta por 5 adultos (4 mujeres y 1 hombre) con edades comprendidas entre los 64 y 74 años, con un nivel de estudio básico. Los criterios de inclusión para el grupo de pacientes EA se establece según un estadio de demencia "inicial" o "pre-demencia" con puntuaciones del Mini-Examen Cognoscitivo (MEC) entre 14-25, puntuaciones en la Escala de Demencia Global (GDS) situados en el estadio 4-5 de deterioro y puntuaciones inferiores a 10 en el Test de Fluidez verbal de Isaacs. 


\section{Instrumentos}

Se administró una versión computerizada, "Cartas" (Palacios, Paíno y Alameda, 2010), basada en la original tarea de la Iowa Gambling Task de Bechara et al. (1994), versión “ABCD”. En la Iowa Gambling Tastk se debe elegir cartas entre cuatro mazos con el objetivo de incrementar las ganancias, cada carta tiene su propia ganancia y pérdida. En la Tabla 1 se describe la programación de la IGT original.

Tabla 1. Programación de ganancias/pérdidas en la Iowa Gambling Task

\begin{tabular}{lcl}
\multicolumn{1}{c}{ Mazo } & Ganancia por carta & \multicolumn{1}{c}{$\begin{array}{c}\text { Probabilidad de Pérdidas } \\
\text { Cantidades }\end{array}$} \\
\hline A (desventajoso) & 100 & $\mathrm{p}=.5-150,-300,-200,-250,-350$ \\
\hline $\mathrm{B}$ (desventajoso) & 100 & $\mathrm{p}=.1-1250$ \\
\hline $\mathrm{C}$ (ventajoso) & 50 & $\mathrm{p}=.5-25-25,-50,-75,-75$ \\
\hline $\mathrm{D}$ (ventajoso) & 50 & $\mathrm{p}=.1-250$ \\
\hline
\end{tabular}

Tabla 2. Programación de ganancias/pérdidas en la Tarea modificada de la Iowa Gambling Task

\begin{tabular}{lcl}
\multicolumn{1}{c}{ Mazo } & Ganancia por carta & \multicolumn{1}{c}{$\begin{array}{c}\text { Probabilidad de Pérdidas } \\
\text { Cantidades }\end{array}$} \\
\hline A (desventajoso) & 20 & $\mathrm{p}=.5-40,-50,-70$ \\
\hline $\mathrm{B}$ (desventajoso) & 20 & $\mathrm{p}=.1-160$ \\
\hline $\mathrm{C}$ (ventajoso) & 10 & $\mathrm{p}=.5-5,-5,-10$ \\
\hline $\mathrm{D}$ (ventajoso) & 10 & $\mathrm{P}=.1-20$ \\
\hline
\end{tabular}

Se modifica la IGT pensando en las características que presentan los pacientes con EA, se ha reducido el número de elecciones, pasando de 100 a 60 , ya que la tarea se hace larga y tediosa para ellos. Al reducir el número de elecciones hemos cambiado la amplitud de los ciclos y de los bloques, mantenemos 5 bloques pero con 12 elecciones en lugar de 20. En este caso los ciclos no son por cada 10 elecciones sino por cada 6, así cabe esperar una mejor captación de la secuencia implícita en cada uno de los mazos. Por último hemos rebajado la cuantía de los premios y castigos, manteniendo la misma línea que en la IGT original. Así, A y B son los mazos desfavorables, frente a C y D que son los mazos favorables. En A y $\mathrm{C}$ se producen 3 pérdidas por cada ciclo y en $\mathrm{B}$ y $\mathrm{D}$, hay una única pérdida. En los mazos desfavorables las ganancias son de $20 €$ por jugada y las pérdidas son de $160 €$ en cada ciclo, lo que supone unas pérdidas de $40 €$ por ciclo. En cambio, en los mazos favorables, las ganancias son de $10 €$ por jugada y $20 €$ de pérdidas por ciclo, lo que supone unas ganancias de $40 €$ en cada ciclo.

El índice gambling (IG) se obtiene de la substracción a la suma del número de elecciones de los mazos ventajosos a la de los desventajosos, es decir $\mathrm{IG}=(\mathrm{C}+\mathrm{D})-(\mathrm{A}+\mathrm{B})$. Los análisis se basan en el IG total, que supone la puntuación en las 60 jugadas, también se determina el desarrollo de las elecciones realizadas en los 5 bloques, compuesto por 12 jugadas cada bloque: B1 (1-12), B2 (13-24), B3 (25-36), B4 (37-48) y B5 (49-60) y la 
exploración del número de elecciones en cada montón y en cada mazo (desventajosos y ventajosos), por bloques.

Mini-Examen Cognoscitivo (MEC) (Lobo et al., 1979). Es una adaptación modificada al español del Mini-Mental State Examination (MMSE) (Folstein, Folstein y McHugh, 1975) que evalúa de forma breve el nivel cognitivo. La puntuación total tiene un rango entre 0 y 35 puntos. Puntuaciones de 23 o menos son indicadores de demencia tipo Alzheimer.

Test del Reloj ‘a la orden' (TRO) y ‘a la copia' (TRC) (Cacho et al., 1999): Se utiliza para la detección de un deterioro global en demencias. La tarea consiste en solicitar al participante que dibuje la esfera de un reloj, con sus manecillas marcando las once y diez. Se evalúan tres aspectos de la ejecución del reloj: esfera, números y manecillas en dos condiciones 'a la orden' y 'a la copia'.

Escala de Deterioro Global (GDS) (Reisberg et al., 1982): Delimita la evolución del deterioro cognitivo en 7 estadios, desde ausencia de deterioro cognitivo (nivel 1) hasta demencia severa (nivel 7).

Isaacs Set Test (IST) (Isaacs y Akhtar, 1972): Mide la fluidez verbal de tipo categorial al solicitar al sujeto que cite en un tiempo limitado (60 segundos) una lista de palabras pertenecientes a categorías semánticas específicas, como nombres de vegetales, animales, frutas, colores y ciudades. Se contabilizaron las respuestas de la subescala animales.

Para la evaluación se han utilizado los siguientes instrumentos:

1. Cuestionario sociodemográfico, elaborado específicamente para esta investigación, en el que se registran las variables personales y demográficas.

2. Inventario de Estilos de Personalidad de Millon (MIPS-Millon, 1994). Adaptación española de Sánchez López, Díaz Morales y Aparicio García (2001). Consta de 24 escalas de personalidad agrupadas en 12 pares y distribuidas en tres áreas: Metas Motivacionales, Modos Cognitivos y Conductas Interpersonales. También consta de tres escalas de validez de respuestas y uno de ajuste: Impresión Positiva (IP), Impresión Negativa (IN), Consistencia (C) y el Índice de Ajuste (Iaj) que mide el grado de adaptabilidad de la persona. La información sobre el procedimiento utilizado para la adaptación del MIPS a la población española, así como sobre sus propiedades psicométricas para esta población, está publicada en la adaptación española (Millon, 2001)

\section{Procedimiento}

Se evaluó de forma individualizada el grado de deterioro cognitivo a través de varias pruebas: El Miniexamen Cognoscitivo de Lobo (MEC) (Lobo, Ezquerra, Gómez, Sala y Seva, 1979); el Test del Reloj (Cacho, García-García, Arcaya, Vicente y Lantada, 1999); el Test de Fluidez Verbal de Isaacs (Isaacs y Akhtar, 1972) mediante la subescala 
animales y también evaluados en la Escala de Deterioro Global (GDS) (Reisberg, Ferris, de Leon y Crook, 1982). Para posteriormente, medir la toma de decisiones mediante la versión reducida y modificada de la IGT, denominada "Cartas" (Palacios, Paíno y Alameda, 2010).

\section{Análisis de datos}

Mediante la prueba $t$ de Student para muestras independientes se analizaron las diferencias de medias entre los grupos para la edad y para las pruebas de cribado (MEC, IST, GDS, TRO, TRC). Los dos grupos se han comparado mediante un modelo lineal general de medidas repetidas para ver las diferencias del IG durante los cinco bloques de la tarea, se realizó un diseño 2 x 5 con un factor intersujeto (Grupo) y otro intrasujeto (Bloques, con 5 niveles). El número de cartas seleccionadas en cada uno de los montones (A, B, C y D), fue analizado con una $t$ de Student para muestras independientes y relacionadas. Un diseño factorial de medidas repetidas 2 x 2 x 5, con un factor intersujeto (Grupo), y dos factores intrasujeto; Mazos con dos niveles (mazos ventajosos y desventajosos) y Bloques con cinco niveles, fue realizado para comprobar las diferencias en el número de elecciones de cada mazo en los diferentes bloques de la tarea. Para determinar las correlaciones entre las pruebas de cribado y el rendimiento en la IGT se realizó una correlación de Pearson.

\section{RESULTADOS}

Cinco sujetos componen el grupo de pacientes con enfermedad tipo Alzheimer en fase leve ( 1 hombre y 4 mujeres), con una edad media de 69,60 años $(D T=4,03)$ y 5 participantes pertenecientes al grupo control ( 3 mujeres y 2 hombres), con una edad media de 76,80 (DT=9,26). No se hallan diferencias estadísticamente significativas entre los dos grupos para la variable edad $(p>, 05)$. En la Tabla 3 se muestran los datos correspondientes a las pruebas de cribado para la detección de demencias, observándose diferencias significativas entre el grupo de pacientes y el grupo control en el $\operatorname{MEC}\left(t_{(1-8)}=-3,13 ; p<, 05\right)$ y $\operatorname{GDS}\left(t_{(1-8)}=4,110 ; p<, 05\right)$.

Tabla 3. Media (DT) de las pruebas de cribado para la detección de demencias en cada grupo

\begin{tabular}{cccc}
\hline \multirow{2}{*}{ Pruebas } & Pacientes DTA & Controles & \multirow{2}{*}{$p$} \\
\cline { 2 - 4 } & Media (DT) & Media (DT) &, $014^{*}$ \\
\hline MEC & $21,60(4,50)$ & $30,40(4,39)$ &, 98 \\
\hline IST & $7,60(2,79)$ & $12,60(5,27)$ &, $003^{*}$ \\
\hline GDS & $4,4(, 55)$ & $1,90(1,24)$ &, 931 \\
\hline TRO & $8,6(1,95)$ & $8,5(1,54)$ &, 298 \\
\hline TRC & $8,8(1,79)$ & $9,7(, 27)$ & \\
\hline * Diferencias significativas $(p<, 05)$ & & \\
\hline
\end{tabular}


La puntuación media obtenida por el grupo de pacientes en el IG total fue de $-3,60(D T=3,29)$, mientras que el grupo control obtuvo una puntuación media de $4(D T=3,16)$, siendo esta diferencia significativa $\left(t_{(1-8)}=-3,73 ; p<, 01\right)$.

Para más precisión sobre la evolución de la prueba y sus curvas de aprendizaje se establecieron los análisis correspondientes para los cinco bloques de ensayos de la tarea. La puntuación gambling media obtenida por los bloques, tanto en pacientes como en el grupo control se observa en la Tabla 4. Observándose diferencias significativas entre los grupos en el Bloque $4\left(t_{1-8}=-2,68 ; p<, 05\right)$.

Tabla 4. Media (DT) en los bloques de ensayos en cada grupo

\begin{tabular}{lccc}
\hline \multicolumn{3}{c}{ Promedio de IG en cada grupo para cada bloque de la tarea } \\
\cline { 2 - 4 } Bloques & Pacientes DTA & Controles & \multirow{2}{*}{ Media (DT) } \\
\cline { 2 - 4 } &,$- 40(, 89)$ & Media (DT) &, 373 \\
\hline B1 (1-12) & $-1,60(1,67)$ &, $80(1,79)$ &, 060 \\
\hline B2 (13-24) & $-1,20(5,39)$ & $1,20(1,79)$ &, 101 \\
\hline B3 (25-36) & $-1,60(, 89)$ &, $80(1,79)$ &, $028^{*}$ \\
\hline B4 (37-48) & $1,20(1,1)$ &, $80(1,79)$ &, 681 \\
\hline B5 (49-60) & & \\
\hline * Diferencias significativas $(\mathrm{p}<0,05)$ & & \\
\hline
\end{tabular}

En la Figura 1, se representan estas puntuaciones en los cinco bloques de la tarea para poder apreciar la evolución del rendimiento. Los datos determinan que no existen diferencias significativas para la interacción entre los factores grupo y bloque $\left(F_{(1-32)}=1,468 ; \mathrm{p}>, 05\right)$.

Figura 1. Gráfica del rendimiento del IG por bloques en cada grupo

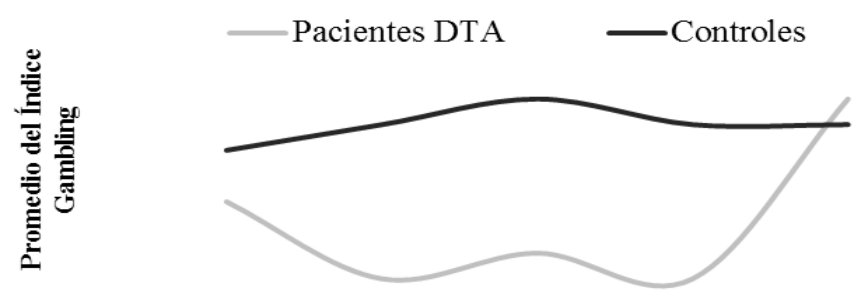

Bloques

Las elecciones en cada grupo para cada montón (A, B, C y D) son las siguientes; el grupo de pacientes obtuvieron una media de 12,60 (DT=2,61) elecciones en el mazo A, de 19,20 (DT=3,35) en el mazo B, de 15,80 (DT=2,77) en el mazo C y por último, $12,40(D T=3,29)$ en el mazo D y el grupo control obtuvo $12,20(D T=4,02)$ en el mazo A, $15,80(D T=3,11)$ en el mazo B, 15,60 (DT=2,07) en el mazo C, y 16,40 
$(D T=1,52)$ en el mazo D. Estos datos muestran que el grupo control eligió con mayor frecuencia los mazos B y D y el grupo de pacientes con mayor frecuencia los mazos B y C. Se observan diferencias significativas entre ambos grupos en el número de elecciones en el montón D $\left(t_{(1-8)}=-2,471 ; p<, 05\right)$ (ver Figura 2$)$.

Figura 2. Promedio del número de elecciones en cada mazo en cada grupo

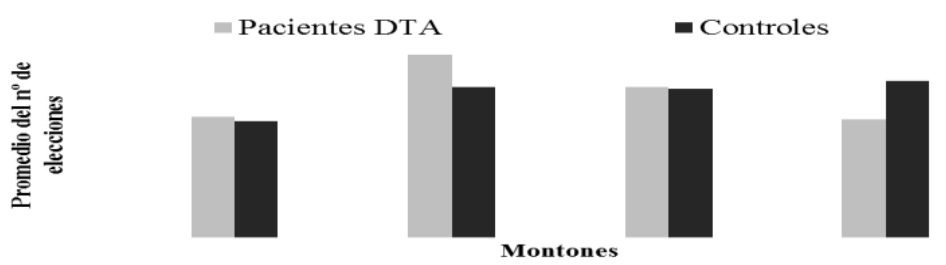

Los datos sólo muestran diferencias significativas, a nivel intrasujeto, entre los mazos B-C en el grupo de pacientes DTA $\left(t_{(1-4)}=3,666 ; p<, 05\right)$.

Se determinó las diferencias existentes en función del tipo de carta, ventajosas o desventajosas (Tabla 5) y por bloques, en una triple interacción se obtienen diferencias significativas entre el factor mazo, bloque y grupo $\left(F_{(4-32)}=5,560 ; p<, 01\right)$. Se observan diferencias significativas en los mazos desventajosos $\left(t_{(1-8)}=3,726 ; p<, 01\right)$ y ventajosos $\left(t_{(1-8)}=-3,726 ; p<, 01\right)$ entre ambos grupos, si bien, el grupo de pacientes con enfermedad de Alzheimer tiene un mayor número de elecciones desventajosas y el grupo control un mayor número de elecciones ventajosas.

Tabla 5. Media (DT) de las elecciones en los mazos desventajosos y ventajosos para cada bloque en cada grupo

\begin{tabular}{|c|c|c|c|}
\hline \multirow{2}{*}{ Mazos } & \multirow{2}{*}{ Bloques } & Paciente DTA & Controles \\
\hline & & Media (DT) & Media (DT) \\
\hline \multirow{6}{*}{ VENTAJOSOS } & B1 (1-12) & $5,80(, 45)$ & $6,20(, 84)$ \\
\hline & B2 (13-24) & $5,20(, 84)$ & $6,40(, 89)$ \\
\hline & B3 (25-36) & $6,60(1,14)$ & $5,40(, 89)$ \\
\hline & B4 (37-48) & $6,80(, 45)$ & $5,60(, 89)$ \\
\hline & B5 (49-60) & $5,40(, 55)$ & $5,60(, 89)$ \\
\hline & Total & $28,20(1,64)$ & $32(1,58)$ \\
\hline \multirow{6}{*}{ DESVENTAJOSOS } & B1 (1-12) & $6,20(, 45)$ & $5,80(, 84)$ \\
\hline & B2 (13-24) & $6,80(, 84)$ & $5,60(, 89)$ \\
\hline & B3 (25-36) & $5,40(1,14)$ & $6,60(, 89)$ \\
\hline & B4 (37-48) & $5,20(, 45)$ & $6,40(, 89)$ \\
\hline & B5 (49-60) & $6,60(, 54)$ & $6,40(, 89)$ \\
\hline & Total & $31,80(1,64)$ & $28(1,58)$ \\
\hline
\end{tabular}

Las diferencias existentes entre ambos grupos se establecen en las elecciones de las ventajosas en el B4 $\left(t_{(1-8)}=2,689 ; \mathrm{p}<, 05\right)$ y las diferencias son significativas entre 
los grupos en el B4 $\left(t_{(1-8)}=-2,683 ; \mathrm{p}<, 05\right)$ de las elecciones desventajosas. En el grupo de pacientes DTA existen diferencias significativas para la doble interacción entre el factor mazo y el factor bloque $\left(F_{(4-16)}=4,704 ; \mathrm{p}<, 05 ;\right)$. En pacientes con EA, las diferencias entre los bloques de los mazos ventajosos se establecen entre : B1-B4 $\left(t_{(1-4)}=-3,162\right.$; $\mathrm{p}<, 05)$, B2-B4 $\left(t_{(1-4)}=-6,532 ; \mathrm{p}<, 05\right)$ y B4-B5 $\left(t_{(1-4)}=5,715 ; \mathrm{p}<, 05\right)$, y estas mismas diferencias significativas se identifican en el mazo desventajoso entre los bloques: B1B4 $\left(t_{(1-4)}=3,162 ; \mathrm{p}<, 05\right), \mathrm{B} 2-\mathrm{B} 4\left(t_{(1-4)}=6,532 ; \mathrm{p}<, 01\right)$ y B4-B5 $\left(t_{(1-4)}=-5,715 ; \mathrm{p}<, 01\right)$. Los únicos datos, sobre las diferencias en cada bloque entre los mazos desventajosos y ventajosos para aquellos que padecen la enfermedad de Alzheimer, son diferencias significativas en el B4 $\left(t_{(1-4)}=4 ; \mathrm{p}<, 05\right)$. Para el grupo control no se hayan diferencias significativas entre la interacción del factor mazo con el factor bloque $\left(F_{(4-16)}=1,469\right.$; p>,05) (Ver Figura 3).

Figura 3. Promedio del número de elecciones en los mazos ventajosos y desventajosos por bloques en la IGT. A la izquierda el grupo de pacientes DTA y a la derecha el grupo control
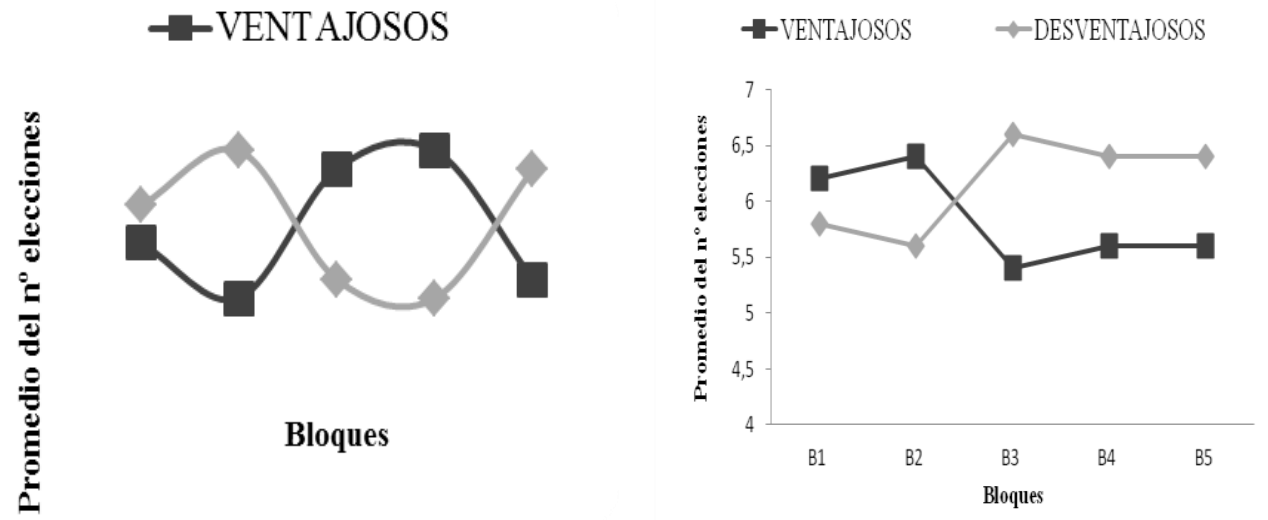

Tras la realización de una correlación de Pearson entre las diferentes pruebas de cribado y la ejecución en la IGT, se observan correlaciones significativas y relativamente elevadas entre; MEC y IST $(r=, 827$; p<,05); MEC y GDS ( $r=-, 933$; $\mathrm{p}<, 001)$ y GDS con IST $(r=-, 774 ; \mathrm{p}<, 05)$. No se establece ningún tipo de correlación entre la IGT y las pruebas de cribado $(\mathrm{p}>, 05)$.

\section{DISCUSIÓN}

Hemos intentado estudiar el proceso de toma de decisiones a través de una tarea modificada de la IGT, para ello hemos analizado la ejecución de un grupo de 5 pacientes con DTA y 5 controles de similares características. Los primeros datos a destacar son las diferencias observadas en las pruebas cognitivas (MEC y GDS) lo que 
pone de manifiesto las diferencias entre ambos grupos (Tabla 3), además hemos comprobado que no hay correlación entre la IGT y los test de cribado de demencias.

También observamos diferencias significativas en cuanto al IG, lo que nos pone de manifiesto una peor ejecución global de los pacientes, coincidente con los datos aportados en otras investigaciones (Delazer et al., 2007; Moreno y Alameda, 2011; Zamarian et al., 2011). Sin embargo, en el análisis por bloques sólo se obtiene diferencia significativa entre ambos grupos en el bloque 4 (Tabla 4; Figura 1), lo que pone de manifiesto que la evolución de la misma no difiere mucho en ambos grupos, de hecho en el último bloque las puntuaciones IG de ambos grupos son similares, y no existen diferencias significativas para la interacción entre los factores grupo y bloque. En cuanto a las elecciones de cartas, se puede apreciar (Figura 2) que hay coincidencia en los mazos A y C, y diferencias en $\mathrm{B}$ y D, siendo el mazo B el más elegido por los pacientes, en cambio en los controles, B, C y D presentan niveles similares de selección, lo que pone de manifiesto un fenómeno ampliamente observado en la IGT, los problemas para identificar B como un mazo desfavorable, ya que su elección parece estar más relacionado con el número de pérdidas que con la cuantía total de las mismas , esta preferencia por el mazo B en los pacientes DTA explica la preferencia por los mazos desventajosos, frente a los controles, siendo más evidente en el bloque 4 .

La ejecución de ambos grupos es relativamente próxima, y si tenemos en cuenta las diferencias en los indicadores cognitivos, esto nos puede hacer pensar la independencia del proceso de toma de decisiones de las variables cognitivas medidas, al tiempo que dadas las bajas puntuaciones de los sujetos control, nos hace pensar que los déficits en la toma de decisiones no están relacionados directamente con el deterioro cognitivo (Bechara et al., 2001; Dunn, Dalgleish y Lawrence, 2006; Anderson Jhonson et al., 2008). Por otra parte, con esta modificación de la IGT se minimizan las diferencias observadas entre ambos grupos, por lo que podemos pensar que nuestra tarea mejora el rendimiento de los pacientes, si bien esta afirmación necesita ser investigada a fondo.

\section{REFERENCIAS}

Anderson, S.W., Bechara, A., Damasio, H., Tranel, D. y Damasio, A.R. (2002). Impairment of social and moral behavior related to early damage in human prefrontal cortex. Foundations of Social Neuroscience, 333-343.

Anderson, C., Xiao, L., Palmer, P., Sun P., Wang, Q., Wei, Y., Jia, Y., Grenard, J., Stacy, A. y Bechara, A. (2008). Affective decision-making deficit, linked to a dysfunctional ventromedial prefrontal cortex, revealed in 10th grade Chinese adolescent binge drinkers. Neuropsychologia, 46, 714-726.

Bark, R., Dieckmann, S., Bogerts, B. y Northoff, G. (2005). Deficit in decision making in catatonic schizophrenia: an exploratory study. Psychiatry Research, 134(2), 131-141. 
Bechara, A., Damasio, A.R., Damasio, H. y Anderson, S.W. (1994). Insensitivity to future consequences following damage to human prefrontal cortex. Cognition, 50(1-3), 7-15.

Bechara, A., Damasio, H. y Damasio, A.R. (2000). Emotion, decision making and the orbitofrontal cortex. Cerebral Cortex, 10(3), 295-307.

Bechara, A., Damasio, H., Tranel, D. y Damasio, A.R. (1997). Deciding advantageously before knowing the advantageous strategy. Science, 275(5304), 1293-1295.

Bechara, A., Dolan, S., Denburg, N., Hindes, A., Anderson, S.W. y Nathan, P.E. (2001). Decision-making deficits, linked to a dysfunctional ventromedial prefrontal cortex, revealed in alcohol and stimulant abusers. Neuropsychologia, 39(4), 376-389.

Bechara, A., Tranel, D., Damasio, H. y Damasio, A.R. (1996). Failure to respond autonomically to anticipated future outcomes following damage to prefrontal cortex. Cerebral Cortex, $6(2), 215-225$.

Cacho, J., García-García, R., Arcaya, J., Vicente, J.L. y Lantada, N. (1999). Una propuesta de aplicación y puntuación del test del reloj en la enfermedad de Alzheimer. Rev Neurol, 28(7), 648-55.

Cavedini, P, Riboldi, G., D’Annucci, A., Belotti, P., Cisima, M. y Bellodi, L. (2002). Decisionmaking heterogeneity in obsessive-compulsive disorder: ventromedial prefrontal cortex function predicts different treatment outcomes. Neuropsychologia, 40(2), 205-211.

Cavedini, P., Zorzi, C., Baraldi, C., Patrini, S., Salomoni, G., Bellodi, L., Freire, R.C. y Perna G. (2012). The somatic marker affecting decisional processes in obsessive-compulsive disorder. Cognitive Neuropsychiatry, 17(2), 177-190.

Clark, L., Manes, F., Antoun, N., Sahakian, B.J. y Robbins, T.W. (2003). The contributions of lesion laterality and lesion volume to decision-making impairment following frontal lobe damage. Neuropsychologia, 41(11), 1474-1483.

Damasio, A.R. (1994). Descartes\& error. New York: Putnam.

Damasio, A.R., Tranel, D. y Damasio, H. (1990). Individuals with sociopathic behavior caused by frontal damage fail to respond autonomically to social stimuli. Behavioural brain research, 41(2), 81-94.

Dante, C. (2006). Patrón de toma de decisiones desventajosas en pacientes drogodependientes. Salud y drogas, 6(1), 71-88.

De Ruiter, M.B., Oosterlaan, J., Veltman, D.J., van den Brink, W. y Goudriaan, A.E. (2012). Similar hyporesponsiveness of the dorsomedial prefrontal cortex in problem gamblers and heavy smokers during an inhibitory control task. Drug and Alcohol Dependence, $121(1-2), 81-89$.

Delazer, M., Sinz, H., Zamarian, L. y Benke, T. (2007). Decision-making with explicit and stable rules in mild Alzheimer's disease. Neuropsychologia, 45(8), 1632-1641.

Dunn, B.D., Dalgleish, T. y Lawrence, A.D. (2006). The somatic marker hypothesis: A critical evaluation. Neuroscience and Biobehavioral Reviews, 30(2), 239-271.

Folstein, M.F., Folstein, S.E. y McHugh, P.R. (1975). «Mini-mental state». A practical method for grading the cognitive state of patients for the clinician. Journal of Psychiatric Research, 12(3), 189-198. 
García-Molina, A., Rodríguez, P., Vendrell, P., Junqué, C. y Roig-Rovira, T. (2008). Disfunción orbitofrontal en la esclerosis múltiple: Iowa Gambling Task. Psicothema, 20(3), 445449.

García-Molina, A., Roig-Rovira, T., Enseñat-Cantallops, A., Sánchez-Carrión, R., Picó-Azanza, N. y Peña-Casanova, J. (2007). Exploración de los procesos de toma de decisiones en pacientes con traumatismo craneoencefálico. Neurología, 22(4), 206-212.

Goudriaan, A.E., Oosterlaan, J., de Beurs, E. y van den Brink, W. (2005). Decision making in pathological gambling: a comparison between pathological gamblers, alcohol dependents, persons with Tourette syndrome, and normal controls. Brain Research. Cognitive Brain Research, 23(1), 137-151.

Grant, S., Contoreggi, C. y London, E.D. (2000). Drug abusers show impaired performance in a laboratory test of decision making. Neuropsychologia, 38(8), 1180-1187.

Isaacs, B. y Akhtar, A.J. (1972). The set test: a rapid test of mental function in old people. Age and ageing, 1(4), 222-226.

Johnson, C.A., Xiao, L., Palmer, P., Sun, P., Wang, Q., Wei, Y., Jia, Y., et al. (2008). Affective decision-making deficits, linked to a dysfunctional ventromedial prefrontal cortex, revealed in 10th grade Chinese adolescent binge drinkers. Neuropsychologia, 46(2), 714-726.

Kleeberg, J., Bruggimann, L., Annoni, J.M., van Melle, G., Bogousslavsky, J. y Schluep, M. (2004). Altered decision-making in multiple sclerosis: a sign of impaired emotional reactivity? Annals of Neurology, 56(6), 787-795.

Lobo, A., Ezquerra, J., Gómez, B.F., Sala, J.M. y Seva, D.A. (1979). Cognocitive mini-test (a simple practical test to detect intellectual changes in medical patients). Actas lusoespañolas de neurología, psiquiatría y ciencias afines, 7(3), 189.

Mazas, C.A., Finn, P.R. y Steinmetz, J.E. (2000). Decision-making biases, antisocial personality, and early-onset alcoholism. Alcoholism, Clinical and Experimental Research, 24(7), 1036-1040.

Moreno, A. y Alameda, J.R. (2011). Demencia tipo Alzheimer, deterioro cognitivo y toma de decisiones. European Journal of investigation in health, psychology and education, $1(1)$.

Palacios, E., Paíno, S.G. y Alameda, J.R. (2010). Programa Cartas. Descargado: http://www.telecable.es/personales/elias.palacios/CartasSetup.jar.

Petry, N.M., Bickel, W.K. y Arnett, M. (1998). Shortened time horizons and insensitivity to future consequences in heroin addicts. Addiction, 93(5), 729-738.

Price, B.H., Daffner, K.R., Stowe, R.M. y Mesulam, M.M. (1990). The comportmental learning disabilities of early frontal lobe damage. Brain: a journal of neurology, 113, 13831393.

Reisberg, B., Ferris, S.H., de Leon, M.J. y Crook, T. (1982). The Global Deterioration Scale for assessment of primary degenerative dementia. The American journal of psychiatry, 139(9), 1136-1139.

Ritter, L.M., Meador-Woodruff, J.H. y Dalack, G.W. (2004). Neurocognitive measures of prefrontal cortical dysfunction in schizophrenia. Schizophrenia Research, 68(1), 65-73. 
Shallice, T. (1982). Specific impairments of planning. Philosophical transactions of the Royal Society of London. Series B, Biological sciences, 298(1089), 199-209.

Tirapu-Ustárroz, J., Muñoz-Céspedes, J.M. y Pelegrín-Valero, C. (2002). Funciones ejecutivas: necesidad de una integración conceptual. Revista de Neurología, 34(7), 673-685.

Tomb, I., Hauser, M., Deldin, P. y Caramazza, A. (2002). Do somatic markers mediate decisions on the gambling task? Nature Neuroscience, 5(11), 1103-1104.

Verdejo, A., Aguilar de Arcos, F. y Pérez-García, M. (2004). Alteraciones de los procesos de toma de decisiones vinculados al córtex prefrontal ventromedial en pacientes drogodependientes. Revista de neurología, 38(7), 601-606.

Zamarian, L., Weiss, E.M. y Delazer, M. (2011). The impact of mild cognitive impairment on decision making in two gambling tasks. The Journals of Gerontology Series B: Psychological Sciences and Social Sciences, 66(1), 23.

Recibido: 29 de febrero de 2012

Recepción Modificaciones: 23 de marzo de 2012

Aceptado: 29 de marzo de 2012 